produces bedsores, and the pressure of an iron splint is unbearable. Moreover, to children of this age perfect rest in one position is the acutest misery. But if this is bad, the next stage is infinitely worse. A large abscess forms in or around the joint, with disintegration of the femoral head or the acetabulum, or both. It is necessary to remove the necrotic or carious bone, and to provide free exit for the abundant pus. At the same time it is imperative that the large cavity produced should be kept aseptic, or septic fever or pyæmia will certainly carry off the patient, already so greatly enfeebled. But the opening which has to be made is at the very centre of the body, and close to the two main emunctories, and the dressing of the wound is painful, and almost necessitates the lifting of the patient, when movements at the very spot which should be most immovable will certainly take place. Sooner than call attention to himself and risk the repetition of the dreaded dressing, the child will lie with its excretions soaking into the coverings of the wound; and it requires the most careful nursing to prevent this. Careful nursing may, and doubtless will, do much to obviate the danger; but it cannot prevent the pain endured at every dressing or cleaning, nor the everrecurrent dread in which the life of the child is passed at this time, when of all others, for the best chance of its recovery, its mind should be at ease. And to add to all this, one of the best helps to the child's recovery is fresh air, and this is almost, if not quite, unobtainable. To dress the wound sufficiently often to secure asepsis without pain or movement at the diseased part, to permit of the performance of the natural functions without danger of soakage of decomposable or decomposed material into the cavity produced by operation, to keep the bones of the trunk and tower limb in their relative position whilst permitting the area of pressure to be varied from time to time, or even, if possible, occasionally allowing the patient the upright position-these are the problems which every surgeon tries to solve who has to treat one of these severe examples of morbus coxæ. And the appliances at present in use do not assist him greatly. Some-most-are effective onjy so long as the pabient lies supine, the bed itself forming an integral part of them; so that the difficulbies arising from decubitus are not to be thus overcome. Some are too heavy, and some must be removed at the time when they are most required, during the change of dressings, To assist in solving the difficulty I have devised a bed which is pivoted, the pivots at each end resting upon upright standards, which with the frarrework beneath form its zupport and hold it at a convenient height from the ground, that the patient shall be on a level coincident with the surgeon's chest. When the child is laid supine, its chest is comfortably held by flexible bands, which are fixed below to the bed itself and united in pairs above. Its legs lie each in a separate channel, which is suitably padded. The sound extremity has its foot firmly braced against a wooden block, which can be fixed at any point, whilst the affected limb has an extension apparatus applied, the cord of which passes through an eyelet on the lower arc, and is connected with a weight, which acts in any position of the bed. The hips xest each upon a pair of trap-doors which open downwards, and allow free access to this part of the body. The traps beneath the sound hip are always bolted, and maintain, in conjunction with the extension, the body and diseased limb always in the same plane, whilst the unsound region can be reached with comfort, dresced or cleaned with ease, and without discomfor's to the patient.

As the bed is pivoted, it is easy to see that it is capable of alterations in position so that the plane of pressure can be constantly varied. This is usually done by turning the bed so that one of the perforations in the upper pivot coin. sides with one of those in the standard, and fixing it at that angle by a steel pin; but if greater range of movement is required, a worm-and-wheel movement is attached, which renders it practically unlimited. In any case, there is no alteration in the relative position of parts operated upon, as the whole body is moved at one time. When the case is sufficiently advanced to permit of the upright position, the lower are and the extension can be removed, and the entire bed placed against the wall in an almost vertical position. The patient then resting his sound foot on the block, and secured to the bed by the chest and hip bands, has extension going on by the weight of the unsound leg, and is in practically the same condition as if he had a Thomas's splint and patten applied. Several severe cases have been treated by its means in the Ancoats
Hospital, and in every instance with success, whilst the comfort to nurses and surgeon, and the freedom from the distressing scenes formerly so common, have been most marked. But whilst this bed was originally intended for the treatment of severe morbus coxa, it is extremely useful for other purposes, such as the treatment of fractures of the lower extremity, and for any case in which the supine position has to be maintained for any length of time.

These beds are now made by John Carter, 6A, New Cavendish -street, Portland-place, W.

Manchester.

\section{LARYNGEAL CHOREA.}

\section{BY JAS. H. NICOLL, M.B.,}

SURGEON TO OUT-PATIENTS, GLASGOW WESTERN INFIRMARY, ETC

BY the term "Iaryngeal chorea" is here meant an affection of the laryngeal muscles similar to that of the other muscles of the body in the disease commonly described as chorea. Under the term "laryngeal chorea," or "chorea of the larynx," certain authors have described an affection of the laryngeal muscular system resulting in certain defects in vocalisation. The descriptions of its symptoms and causation are almost as varied as the anthors re. ferring to it are numerous. It would seem to be in some respects analogous to "writer's cramp,"-i.e., a fatigue or spasm of certain of the laryngeal muscles, and the latest view of its causation seeks to trace it to a fanlty habit of voice production from an inability to breathe during sustained speech or song. The term "vocal asynergy" has been given to it, and, both for the sake of distinction and because the term seems more accurately descriptive, it would be well to use it alone.

That the laryngeal muscles are liable, in common with all the muscles of the body, to suffer in chorea and to exhibit the movements characteristic of that disease is well known. Ziemssen and other authors have described such movements of the laryngeal and respiratory muscles, with resulting irregularities in speech and respiration ard the production of unexpected "grunting" noises in the throat. During the past four years there have come under my observation three cases of chorea commencing in the pharyngeal and laryngeal muscles, and limited for some considerable time to these muscles, the only symptom being a cough. As these cases, in the first place, presented very considerable difficulty in diagnosis, and, in the second place, are of a class to which I have been able to find only one distinct reference in the more accessible literature of chorea, and as, finally, they came under my notice accidentally, I feel constrained to give them publicity in order that the value of the observations made, and the conclusions drawn therefrom, may be tested by those who, from the nature of their practice, have more ample means of observation of choreic patients and of laryngeal disorders. That such cases are fairly common seems probable, unless one is to conclude that my experience has been altogether exceptional. That the true diagnosis of the laryngeal affection, as evidenced by the cough, is frequently missed seems still more probable, seeing that a laryngoscopic examination is necessary to establish it, such an examination being by no means of easy accomplishment in children, the usual victims of chorea. In Case 1 the correctness of the diagnosis may be questioned, but as it was the means of directing inquiry in the second and third cases I give it here along with them.

CASE 1 -J. H- aged eight. This boy is the son of a lay friend in Glasgow. Four years ago, when house surgeon in Glasgow, and visiting frequently at my friend's honse, my attention was directed by the parents to the boy's condition, which was giving them some anxiety. At that time be was a healthy well. grown child of nearly four, and had had measles only of the usual troubles of childhood. There was neither family nor personal history of rheumatism or any "neurosis," nor had he suffered from fright or shock. For several months he had been troubled with a cough, which distressed both himself and his parents, and for which he had been unsuccessfully treated by several practitioners. The cough, which at first had recurred at intervals of hours, had gradually increased in frequency of repetition to intervals 
of only a few minutes towards night, though in the early part of the day the intervals were more prolonged. It always seased during sleep, though not infrequently preventing sleep for a time after the child was put to bed. I did not see the child professionally, and therefore did not examine him in any way, but the characteristics of the cough were unusual in a child, and attracted my attention. Nasal respiration was free, and there was no abnormal respiratory sound. The cough was invariably of the same type. It was not the hoarse or aphonic cough of laryngeal inflammation or growth, nor the noisy "moist" rattling or wheezing cough of bronchitis. It had not the prolonged paroxysms and inspiratory crow of pertussis, nor the "hawking," "hemming " character of the cough due to the effort to clear secretion from the pharynx. It consisted in a sudden single sharp dry cough, like that produced in adults by the sharp contact of a probe with the external auditory meatus near the tympanic membrane. It appeared, in short, to be the cough of momentary irritation of some spot capable of reflexly exciting the cough centre. The cough persisted, gradually increasing in fre. quency, for ten months, when typical choreic movements appeared in the legs, gradually extending over the entire body, with great increase in the severity of the cough, which became still more frequent and of a hoarser quality. The attack of general chorea lasted over one year, was treated by bromides, and since its cessation has not returned. The cough diminished with the other symptoms, its final cessation preceding that of the limb movements by several weeks.

CASE 2.-J. S-, aged fourteen, came under my observation at the Public Dispensary in Stoke Newington, London, in November, 1889. He complained of a troublesome, "hacking cough." The characters of the cough were such as to at once recall to my mind the case of my friends' child (Case 1). The description of the one cough exactly describes the other, with the exception that J. S 's mother stated that he frequently coughed during the night. He had had scarlet fever and measles before the age of four. One uncle had chorea in youth. Beyond this there was no history, personal or family, of rheumatism or nervous affection. He was a stunted, thin boy for his years, but was said to enjoy good health. The cough had troubled him for several weeks, and occurred at intervals of two or three minutes. Respiration was normal, and there was slight hoarseness. His lungs and heart, on examination, appeared healthy. On completely stripping him, no muscular tremor or bwitch could be detected in any part. Physical examination of ears and nose detected nothing abnormal, and the mucous membrane of the pharynx and mouth was healthy. The tongue was absolutely free from abnormal movement, but the soft palate exhibited frequent twitchings of momentary duration. Laryngoscopically the ventricular bands seemed of a deeper hue than normal. The vocal cords, while in energetic normal motion, as in phonation and deep inspiration, acted readily and fairly steadily; but when relieved from conscious voluntary effort, and left to the more passive movements of quiet respiration alone, they exhibited frequent jerky movements of unequal extent towards and away from the middle line. So far as could be observed, the two cords were equally affected, and moved simultaneously in all such movements with one exception. Near the junction of the posterior third with the anterior two-thirds of the right cord there was a darkish red area. At intervals the right cord exhibited a movement by which it became partially kinked, the apex of the kink, which was convex outwards, being situated at the red part. (Snch a movement in a vocal cord is something unique, in at least my experience, and its causation opens a wide field for speculation. The only thing approaching it I have met was a case I observed over two years ago, when assistant to the Central London Throat Hospital, in which an elderly man came complaining of his voice "going off in squeaks." In his case, on attempting phonation for low notes, both of his cords were observed, just after sound pro. duction had commenced, to suddenly leave the middle line and jerk oubwards, momentarily forming sharp curves with the convexity outwards. In this case both cords were bent or curved and the position was less of a sharp kink than was the case in $m y$ choreic patient, in whom the kink in the right cord was a more or less angular one, the apex corresponding with, and presumably causing, the small congested area. As to the mechanism by which such a position of the cord became possible, adductor paralysis, which in its early stage at least gives rise to an outward bend during deep inspiration, may be dismissed. The bend was too acute and too transitory, and there was no other evidence of paralysis. It is open to assume either, on the one hand, a sudden spasm of the whole or of a part of the thyro-arytenoid muscle of the affected side; or, on the other, what seems more probable, some sudden tilting or shifting of the thyroid cartilage by the intrinsic or extrinsic muscles, causing some alteration in its relations to the cricoid other than that normally produced by the action of the cricothyroid muscles-a partial dislocation, in fact, or sliding of the inferior cornu of the thyroid on the flat articnlar surface of the cricoid.) The larynx as a whole appeared not to be shifted in position by any choreic spasm of the external muscles attached to it, and, indeed, except in the palate and intrinsic laryngeal muscles, no choreic movements could be detected. The cough exhibited all the reflex associated muscular action normal to such an act, the contemporaneous fixing of the muscles of the neck and the diaphragm, the contraction of the chest and the explosive passage of air through the rima glottidis denoting a physiological combination of forces to effect one object, strongly contrasting with the irregular and apparently purposeless choreic laryngeal movements. On the patient's second visit a week later, in order to obtain a more complete examination, the fauces and pharynx were painted and the larynx sprayed with a 5 per cent. solution of cocaine. (N.B. Much of the information detailed above as to laryngeal movements in this case was obtained at bhe second and subsequent examinations under cocaine.) Within a few minutes af ver the application the cough had entirely ceased. The choreic movements were quite unaffected. The application of cocaine was repeatedly made within the next few weeks, and invariably with the same result-viz., the complete cessation of the cough, the choreic movements continuing unaffected. On several occasions when no cocaine had been employed I probed different points of the pharynx and larynx, and in particular the congested spot on the right cord, in order to discover, if possible, a reflex spot which might excite the cough. I failed to do so, but cannot regard the result as of any conclusive value, as, in in the first place, I easily excited retching in the pharynx; and secondly, the congested spot, which might reasonably be supposed to be the cause of the cough, was not easily hit, owing to the choreic movements. The patient was treated with chloral and bromides. Five weeks after his first admission a slight twitching in both coraco-brachiales was detected. Shortly after the hands and neck became affected, and subsequently the whole of the upper limbs. The choreic attack was a mild one, and the patient was dismissed well nearly four months after the arms were affected, the laryngeal symptoms being the last to subside. The cough ceased with the laryngeal movements, having survived by six weeks the red congested spot, which disappeared on the commencement of the subsidence of the muscular twitchings.

CASE 3.-A.P - aged fifteen, was admitted to the Public Dispensary, Stoke Newington, London, in the spring of last year complaining of a "dry cough." The patient was a healthy-looking well-grown girl approaching puberty. She had never menstruated, had had one previous attack of chorea, and there was a family history of both chorea and rheumatism. The characters of the cough were similar to those in Case 1. On stripping the patient slight but distinct choreic movements were seen in the forearms and fingers. The tongue was slightly affected, but no movements of the palate could be detected. On laryngoscopic examination irregular movements of the cords towards the middle line were seen to occur frequently. Occasionally one cord appeared to move independently of the other, or, at least, to precede the other in movement, but the motions were so rapid and irregular that accurate observation on this point was impossible. The larynx as a whole was frequently raised and depressed by the extrinsic muscles acting on it, which were also to a certain extent affected by choreic spasm. The mucous membrane of the larynx and pharynx seemed perfectly healthy. Nothing abnormal was found in nose or ears, and the lungs and heart appeared to be normal. The pharynx and larynx were sprayed by cocaine on several occasions, but without any such result as followed the application in Case 2. Both cough and muscular movements remained unaffected. The congh, which occurred at intervals of three or four minutes as a rule, occasionally recurred more frequently, so that during a period of from six to ten seconds four to ten coughs 
might occur. Such periods of greater frequency were of very irregular occurrence, and repeated observation convinced we that their commencement coincided with alterations in position of the whole larynx by the extrinsic muscles, and that the alterations with which they were associated were those in a downward direction. Descent of the larynx was, though by no means invariably, frequently the starting-point of one of these periods of frequency. That it was the cause of these periods was difficult of proof; for while ascent, which was easily produced voluntarily in swallowing, failed to produce increased frequency or to excite the cough in any way, descent to any great extent could not be produced ao will, that connected with the production of deep notes being insignificant in extent in comparison with the ascent of deglutition. The amount of descent produced in this patient, however, by choreic spasm of the depressors of the larynx appeared to be all but, if not quite, as great as the amount of ascent. In order to test the point as far as possible, having placed the patient recumbent with her head hanging well back over a pillow under the neck, I grasped the larynx, and, pressing it well up towards the base of the tongue, held it there as steadily as possible. This manouvre, which I repeated on several occasions, never failed to check all cough for a period which varied from twelve minutes to twenty-eight minutes on different occasions, but on all these occasions cough did return before the larynx was released from its fixed position. During fixation of the larynx in such a position laryngoscopic examination was of course impossible, but the movements of the tongue and of the depressors of the larynx continued.

Dr. B. Sachs of New York, writing in the "Encyclopredia of Diseases of Child ren," says : "Laryngeal chorea is a special form of chorea in which the laryngeal muscles are chiefly affected, the resulb being a peculiar bark which is repeated at short intervals. These cases are sometimes mastaken for cases of hysterical bark, but the general restlessness, the age of the patient, and the choreic movements of the tongue make the diagnosis an easy one. From my own experience I infer that this is the rarest form of chorea." This, as I have said, is the only distinct reference to such cases as the above which I have been able to find in the more accessible descriptions of chorea and laryngeal neuroses, and it may be of interest briefly to examine the cases and Dr. Sachs description a little more in detail. In regard to Cases 2 and 3 it seems to be reasonable to conclude that the cough was the resulb of chores of the laryngeal muscles. Case 1 , though very snggestive, has not, for obvious reasons, the same claim. In regard to the ease of diagnosis, I cannot agree with Dr. Sachs. Certainly in Case 3, where there was neither "general restlessness" nor " choreic movements of the tongue," nothing but a laryngoscopic examination could have established a diagnosis, at least for many weeks. Into the interesting question of the mechanism by which the cough was produced in these cases, others with more ample means of observation will be able to enter. Dr. Sachs says: "The laryngeal muscles (vide supra) are chiefly affected, the result being a peculiar bark \&c.," just as, presumably in the same way, in chorea of the forearm the flexors and extensors of the fingers are affected, the result being movements of the fingers. It seems needless to point out that a cough is a purposive act comprising many complicated muscular efforts coördinated into one whole, which is set in action by a reflex excitation or by voluntary effort. Such an act is quite unlike the irregular faulty spasmodic muscular movements of chorea which, when occurring in the larynx or pharynx, may, by obstructing the current of air in a more or less passive and accidental way, cause irregular speech or respiration, or even unusual laryngeal sounds-indeed, a cough could not be produced by any action of the laryngeal muscles alone. The facts of the above cases seem to point to the conclusion that the reflex congh was excited by abnormal sensations in the larynx caused by the choreic movements of the muscles. The cessation of the cough under cocaine in Case 2 seems to demonstrate this, for in this case the only abnormal condition found beyond the choreic movements was the congestive spot, which disappeared six weeks before the cough. Further, cough from such irritation as inflammation or congestion of the laryn. geal mucous membrane wonld hardly exhibit such characters as were observed in these cases, but would be more prolonged and piroxysmal, and would almost certainly be accompanied by hoarseness and aphonia, from an early period of its duration. Hoarseness at a late period of chorea of the laryn $x$ may accompany the cough. This occurred in Case 1 , and was probably due to some amount of conges. tion or inflammation of the laryngeal mucous membrane due to the irritation of the continued cough. The momentary choreic contractions of the muscles would, in all probabiliby, cause unexpected tensions and bulgings, or possibly contacts of various points of a very sensitive mucous membrane, irritation of which invariably leads to cough. The sensations produced in this way would, it may be supposed, simulate closely those due to momentary contact of a foreign body. That in Case 3 cocaine had no effect in checking the sensibility of the membrane and thus stopping the cough is no argument against this view. Stoerck of Vienna and other observers have proved experimentally that cougb may be excited by irritating by contact the mucous mers brane over the inter-ary tenoid folds, the posterior wall 0 the larynx, and the upper surface of the vocal cords, and also the inferior surface of the cords and the trachea, especi ally its posterior wall, for some distance below the laryng. It requires no argument to prove that the application of cocaine, even by the spray, to the inferior surface of the cords and the larynx and trachea below the rima must be a thing of so great difficulty that its possibility is very doubtful. Therefore, should the point irritated by the choreic movements be below the rima, it is not to be expected that the application of cocaine would check the reflex. Now in Case 3 there was (vide supra) some reason to think that the starting point of the reflex was below the rima, for is was just when the larynx descended, and possibly caused some pressure on or folding of some part of the mucous membrane below the cords, that cough was excited or increased. Though experimental demonstration of this was impossible, some negative evidence in proof $a f$ it was obtained by holding the larynx well up under the base of the tongue and jaw as described. This was always successful for a time, but it is open to anyone to argue that tightly grasping the throat would tend for a time to check cough arising from any cause what ever, and that the return of the cough, when presumably the parts had become accustomed to the manual pressure demonstrates this. My own impression is that the amount of compression used was not sufficient to support this argu ment, and that the true explanation is that by preventing descent of the larynx the source of irritation was removed, the ultimate return of the cough being due to one of two causes, either a momentary or gradual relaxation of the restraint on my own part, or an unobserved and graduat movement of the patient to relieve an irksome position by bringing the rest of the body up to the raised larynx, as the larynx could not descend to the body, the effest in either case being the same.

Glasgow.

\section{Clinital aldotes:}

\section{MEDICAL, SURGICAL, OBSTETRICAL, AND THERAPEUTICAL.}

RUPTURED HEART.

By Barrington WM. Mudd, M.B., C.M.Aberd.

As rupture of the heart is a lesion of somewhat rare occurrence, the following case may be of some interest.

J. W-, aged thirty-one, carman, was brought to me a few days ago, having been found ly ing on hisface at his horse's feet quite unconscious. He had been seen a minute or two previously standing at the heads of the horses, ready to back his van into a shed. The men who brought him said they thought the pole of the van must have struck him on the chest, but of course this was mere supposition, as nobody had seen him fall. On admission, half an hour after being picked up, I found him quite unconscious, voluntary motion being absent. He was extremely blanched; pupils widely dilated, and showed no reaction to light; pulse bardly perceptible; perspiration profuse. In this condition he remained for nearly an hour when he began to show signs of slight consciousness.' I asked him if he had any pain; he understood me, and put his hand to his chest. About a quartez 